\title{
What can we learn from a case report? It's not always about the Swiss cheese
}

\author{
Luis G. Quiñonez, MD
}

\author{
From the Department of Cardiovascular Surgery, Boston Children's Hospital, Boston, Mass. \\ Disclosures: Author has nothing to disclose with regard to commercial support. \\ Received for publication Feb 28, 2016; accepted for publication March 1, 2016; available ahead of print March 31, \\ 2016. \\ Address for reprints: Luis G. Quiñonez, MD, Department of Cardiovascular Surgery, Boston Children's Hospital, \\ 300 Longwood Ave, Boston, MA 02115 (E-mail: luis.quinonez@cardio.chboston.org). \\ J Thorac Cardiovasc Surg 2016;152:e27-8 \\ $0022-5223 / \$ 36.00$ \\ Copyright (C) 2016 by The American Association for Thoracic Surgery \\ http://dx.doi.org/10.1016/j.jtcvs.2016.03.015
}

The case report by Price and colleagues ${ }^{1}$ describes the successful use of a donor cardiac graft that had coronary sinus ostial atresia. Graft loss was prevented by recognizing the anomaly, understanding the implications of the defect, imagining a surgical solution, and executing that solution. The authors' solution was to anastomose the left superior vena cava (LSVC), the sole outlet for coronary venous return, to the left atrium.

For the casual reader of the case report, the authors' solution may seem simple and obvious; however, to appreciate the effort, one must think about "what went right" in the system that led to a successful outcome and the possible preconditions that might have contributed to the outcome.

Although the authors provide few details on the sequence of events, it is worth thinking about how this case may have evolved in real time to eventual execution of the surgical solution. The harvesting team recognized the presence of an LSVC, then identified the atretic coronary sinus ostia. The relevant precondition here is the knowledge of congenital heart disease. This case illustrates the importance of recognizing uncommon anatomic congenital variants, and of understanding the anatomic and physiological implications of these anomalies. Cardiac surgeons might not recognize congenital abnormalities if they have never heard about them, read about them, or encountered them. The only way to ensure awareness, education, and exposure to these lesions is to maintain a component of congenital cardiac surgery in the curriculum that leads to certification. In an environment of shorter congenital rotations, restricted duty hours, and limited interest on the part of some trainees, it behooves congenital cardiac surgeons to make the subject matter relevant to trainees whose interest is the practice of adult acquired cardiac surgery.

After recognizing the coronary sinus ostial atresia, the transplantation team understood the implications of the lesion. Once again, the precondition is knowledge of congenital heart disease. This case reminds us that even an imperfect heart can serve a needy donor well. Given

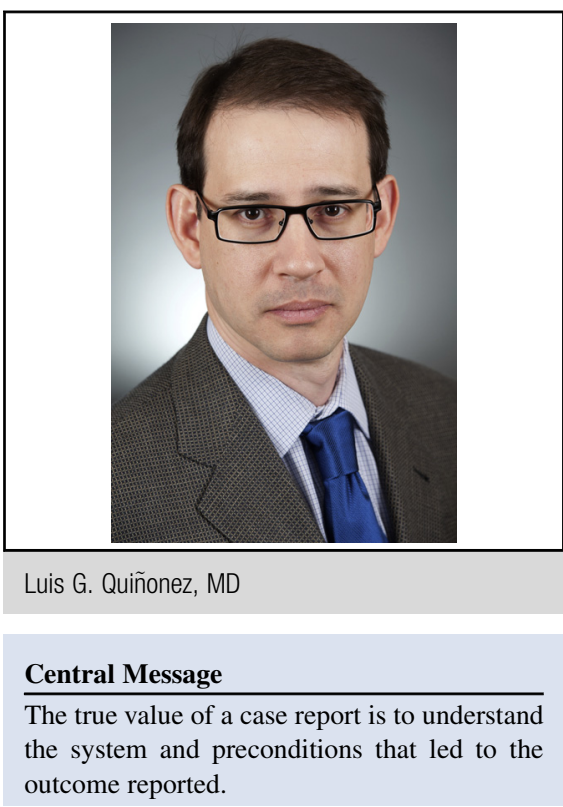

See Article page e25.

the lack of donors, it is important to recognize lesions that may be amenable to immediate surgical correction and likely not affect the long-term outcome of the graft. These organs should not be rejected.

The transplantation team was able to imagine a solution to the problem of coronary venous outflow. We see how innovation can occur directly and immediately at the bedside in the operating room. Innovation is not solely the purview of researchers in laboratories, clinical trials, and translational research programs. Innovation belongs, and is the responsibility of, clinicians at the bedside in both academic and nonacademic settings. This case report is a good example of how to think differently about a clinical problem in an individual patient and come up with an innovative solution. A professional and organizational structure and culture that promotes and allows for such innovation is a necessary precondition.

The transplantation team finally executed the operation they imagined: LSVC to LA anastomosis. One has to appreciate the difficult circumstances under which this all occurred. The team was likely fatigued, working at night with very little sleep after having performed a difficult redo ventricular assist device explant. They were under pressure to implant a heart in a patient with very few other options, using a limited resource and an imperfect donor 
heart, with the stress of an uncertain outcome. Furthermore, there existed the inescapable time pressure of donor heart ischemic time. These adverse preconditions could have led to failure at any point in the chain of events.

When reading case reports, we often see the end result and seldom consider all of the circumstances that led to the reported outcome, positive or negative. The true value of case reports such as that by Price and colleagues ${ }^{1}$ is to understand the system that led to the seemingly simple solution to a rare and potentially life-threatening problem. We often analyze our adverse outcomes by trying to figure out "what went wrong" in the system. Perhaps we should look at our positive outcomes and figure out "what went right." We may learn just as much. It's not always about the Swiss cheese. ${ }^{2}$

\section{References}

1. Price J, Galindez N, Peltz M. Management of coronary sinus ostial atresia in a donor heart during cardiac transplantation. J Thorac Cardiovasc Surg. 2016; 152:e25-6.

2. Wiegmann DA, Shappell SA. A Human Error Approach to Aviation Accident Analysis: The Human Factors Analysis and Classification System. Aldershot, UK: Ashgate Publishing; 2003. 